\title{
Uniform Magnetic Field Characteristics Based UHF RFID Tag for Internet of Things Applications
}

\author{
Abubakar Sharif ${ }^{1,2}{ }^{\mathbb{D}}$, Yi Yan ${ }^{1}$, Jun Ouyang ${ }^{1}$, Hassan Tariq Chattha ${ }^{3} \mathbb{D}$, Kamran Arshad ${ }^{4} \mathbb{D}$, Khaled Assaleh ${ }^{4}$, \\ Abdullah Alhumaidi Alotabi ${ }^{5}$, Turke Althobaiti ${ }^{6}{ }^{D}$, Naeem Ramzan ${ }^{7}$, Qammer Hussain Abbasi ${ }^{2, *}$ (D) and \\ Muhammad Ali Imran ${ }^{2}$ (D)
}

check for updates

Citation: Sharif, A.; Yan, Y.; Ouyang, J.; Chattha, H.T.; Arshad, K.; Assaleh, K.; Alotabi, A.A.; Althobaiti, T.; Ramzan, N.; Abbasi, Q.H.; et al. Uniform Magnetic Field

Characteristics Based UHF RFID Tag for Internet of Things Applications. Electronics 2021, 10, 1603. https:// doi.org/10.3390/electronics10131603

Academic Editors: Pablo Escobedo, Mahesh Soni and Nuria López Ruiz

Received: 25 May 2021

Accepted: 30 June 2021

Published: 3 July 2021

Publisher's Note: MDPI stays neutral with regard to jurisdictional claims in published maps and institutional affiliations.

Copyright: (c) 2021 by the authors. Licensee MDPI, Basel, Switzerland. This article is an open access article distributed under the terms and conditions of the Creative Commons Attribution (CC BY) license (https:/ / creativecommons.org/licenses/by/ $4.0 /)$.
1 School of Electronic Science and Engineering, University of Electronic Science and Technology of China (UESTC), Chengdu 611731, China; sharifuestc@gmail.com (A.S.); yiyan11888@163.com (Y.Y.); yjou@uestc.edu.cn (J.O.)

2 James Watt School of Engineering, University of Glasgow, Glasgow G12 8QQ, UK; muhammad.imran@glasgow.ac.uk

3 Electronic Engineering Technology Department, Okanagan College, Kelowna, BC V1Y 4X8, Canada; chattha43@hotmail.com

4 College of Engineering and IT, Ajman University, Ajman AE 346, United Arab Emirates; k.arshad@ajman.ac.ae (K.A.); k.assaleh@ajman.ac.ae (K.A.)

5 Department of Science and Technology, College of Ranyah, Taif University, P.O. Box 11099, Taif 21944, Saudi Arabia; a.alhumaidi@tu.edu.sa

6 Faculty of Science, Northern Border University, Arar 91431, Saudi Arabia; Turke.althobaiti@nbu.edu.sa

7 School of Computing, Engineering, and Physical Sciences, University of the West of Scotland, Paisley PA1 2BE, UK; Naeem.Ramzan@uws.ac.uk

* Correspondence: qammer.abbasi@glasgow.ac.uk

\begin{abstract}
This paper presents a novel inkjet-printed near-field ultra-high-frequency (UHF) radio frequency identification (RFID) tag/sensor design with uniform magnetic field characteristics. The proposed tag is designed using the theory of characteristics mode (TCM). Moreover, the uniformity of current and magnetic field performance is achieved by further optimizing the design using particle swarm optimization (PSO). Compared to traditional electrically small near-field tags, this tag uses the logarithmic spiral as the radiating structure. The benefit of the logarithmic spiral structure lies in its magnetic field receiving area that can be extended to reach a higher reading distance. The combination of TCM and PSO is used to get the uniform magnetic field and desired resonant frequency. Moreover, the PSO was exploited to get a uniform magnetic field in the horizontal plane of the normal phase of the UHF RFID near-field reader antenna. As compared with the frequently-used commercial near field tag (Impinj J41), our design can be readable up to a three times greater read distance. Furthermore, the proposed near-field tag design shows great potential for commercial item-level tagging of expensive jewelry products and sensing applications, such as temperature monitoring of the human body.
\end{abstract}

Keywords: near-field; ultra-high-frequency (UHF); radio frequency identification (RFID); internet of things (IoT); item-level tagging; temperature sensing

\section{Introduction}

Internet of Things (IoT) has been emerging as the third most innovative wave in the information, communication, and technology (ICT) field, after the Internet and computer. However, some collaborative technologies are co-existing, such as radio frequency identification (RFID), to assist IoT for realizing low-cost item-level or things-level inexpensive tagging or sensing [1-3]. The UHF RFID systems are most prevalent due to their low-cost inkjet printable tags, fast reading, and sensing capabilities [4-10]. Compared with the traditional far-field UHF RFID systems, the near-field UHF RFID system is more suitable for item-level tagging [11]. Similarly, the near-field UHF RFID systems are very useful in application scenarios where confined interrogation volume is required. Previously, most of 
the research work was done on flux-type UHF near-field systems, which are quite similar to the HF RFID system. In these designs, the in-phase current loop is used as the reader antenna to produce a uniform magnetic field in the normal plane, and the electrically small ring was adopted as the tag antenna to receive the magnetic energy [12-14].

For practical application, the near-field RFID systems usually require a read distance of about $0-10 \mathrm{~cm}$. As we know, the RFID chip activation determines the reading distance of the whole RFID system. In previous researches, most of them use the commercial button tag as a test label to prove the uniformity of magnetic field in normal determined height. However, the electric-small antenna's energy reception capability is limited by its size, so if we want to achieve an expected height, the transmitting power of the reader antenna should be enhanced. Unfortunately, the in-phase current loop also has an about $2 \mathrm{dBi}$ omnidirectional gain in the azimuth plane. In the case of high transmitting power usage, it is very easy to read other distant tags, which are unwilling for most of the practical application scenarios. So, the tag's energy reception capability should be enhanced. It is worth noting that just increasing the radius of the tag's antenna is not a good way because, when the radius of the antenna increases, the uniformity of the electric-small ring's current and magnetic field will be changed. Moreover, the near-field UHF RFID tag antennas $[15,16]$ get very little attention compared with the reader antenna. Therefore, the design of a near-field tag antenna with uniform magnetic field characteristics, simple structure, and $100 \%$ reading rate is still challenging for many practical applications.

A quadruple loop near-field reader antenna with uniform magnetic field performance was designed in [17]. This quadruple design consists of fan-shaped loops, feeding lines, and vias for connecting feed lines to those loops. The quadruple tag design achieved uniform magnetic field distribution by opposite direction current cancellation effects. However, this tag design is costly and difficult to fabricate due to the intervention of vias. Moreover, this tag design is fabricated on a $0.8 \mathrm{~mm}$ FR4 substrate. In order to overcome previously mentioned drawbacks, and by attaining motivation from [18], we know that a non-closed ring can also be excited by the normal magnetic field $\left(\mathrm{H}_{\mathrm{z}}\right)$.

In addition to Electromagnetic (EM) techniques and solvers, the use of optimization techniques and algorithms are becoming prevalent both in the EM field and antenna design applications. There are several of algorithms used for antenna and RFID tag optimizations such as PSO, Artificial Bee Colony Optimization, and so forth [19-24]. PSO is one of the best mainstream algorithms used for the optimization of antennas and RFID tags. PSO algorithm mimics the behavior of swarm of flying bees. Recently, PSO has been employed in a wide variety of electromagnetic structures and antennas. In Reference [23], a hybrid PSO algorithm was proposed for bandwidth improvement of inverted F (IFA) antenna. Similarly, PSO was employed for gain enhancement of RFID reader antenna [25], and the planning of a RFID-network by identifying mutual interference and mutual coverage of read-zones among the readers [26].

Therefore, in this paper, a novel inkjet-printed logarithmic spiral UHF RFID near-field tag, based on TCM and PSO, is presented. The benefit of the logarithmic spiral structure lies in its inherited uniform and large magnetic field receiving area that can be extended to achieve a higher reading distance. TCM can define the potential radiation capacity of a conductor with inherent structure along with near and far-field characteristics. Since the antennas' receiving and transmitting characteristics are reciprocal, the PSO is used to optimize the antenna structure to have a uniform magnetic field in the normal phase plane. After the radiation structure is determined, a T-match matching circuit is used to meet the conjugate match with RFID chip impedance [27]. The performance of the proposed design was tested by adopting the same chip as used by the commercial tag (J41). Moreover, to get a robust comparison, the same reader antenna was used for tag reading by previously published researches [12].

The proposed tag can be readable from very close proximity. It achieves three times more read distance as compared to frequently-used commercial near field tag (Impinj J41) with $10 \mathrm{dBm}$ transmission power, thereby greatly reducing the pressure on reader antenna 
designers. The proposed tag design is used to demonstrate tagging applications, such as item-level tagging of jewelry items, as shown in Figure 1. Furthermore, since the tag reading mechanism is based on magnetic field characteristics thereby, it can be used in many sensing and tagging applications [27-30] in collaboration with IoT technology, as shown in Figure 1. For IoT technology assistance, a raspberry pi based setup with LoRa IoT Chip HAT was utilized to connect the RFID reader. The LoRa device provides the connectivity using the LoRa gateway, which helps to store data on the IoT cloud. The data from the IoT cloud can be retrieved for illustration purposes using Web Apps.

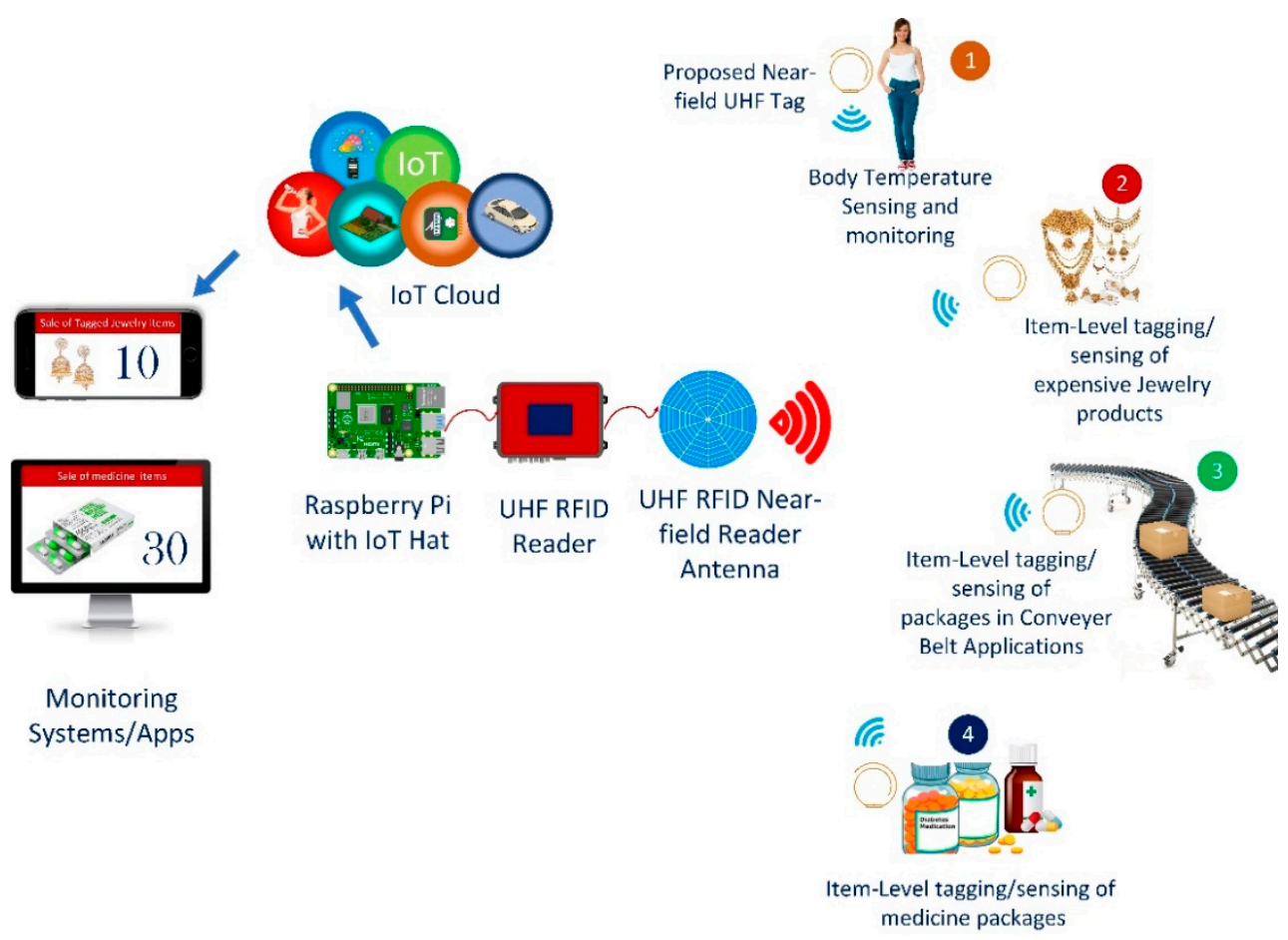

Figure 1. Demonstration of IoT application scenarios using proposed near-field tag/sensor-based IoT and UHF system.

The paper is organized as follows: Section 2 briefly explains the design principle and antenna configuration. Section 3 demonstrates the antenna optimization process using PSO. Section 4 elaborates the results and discusses several performance metrics, such as the read range of the proposed tag design. Moreover, it also discusses jewelry item-level tagging and reading experimental setup using proposed near-field tag design. Finally, conclusions are presented in Section 5.

\section{Design Principle and Antenna Configuration}

In the HF RFID systems, the read distance relates to the size of the tag's coil, which means the bigger size of the tag's coil, the higher the read distance. Whereas, in the UHF RFID systems, just the electrically small loop can meet this condition due to its small wavelength features. Additionally, if the size of the UHF loop tag is increased, the non-uniform and opposite currents will tend to appear.

As mentioned earlier, due to reciprocity property of antennas, the field generated by the same antenna as the receiver is consistent with that generated by the transmitter. If the field distribution of the receiving antenna is consistent with that of the transmitting antenna, the receiving antenna can extract more energy from the field. The higher the consistency between transmitting and receive antennas, the higher the energy captured by the receiving antenna. It is quite similar to the fact that, when the polarization mismatch between transmitting and receiving antennas will be minimum, the associated energy transfer will be maximum. 
Therefore, the structure UHF band near-field tag is not necessarily a closed-loop structure, as long as its near-field magnetic field distribution is consistent with the magnetic field distribution generated by the transmitting coil in space. More precisely, if the field distribution of the mode near-field magnetic field, obtained by the characteristic mode theory analysis, is consistent with the field distribution of the magnetic field in space, the structure has the potential ability to receive energy from the field distribution of the magnetic field.

By TCM theory, we can get physical insight into a metal object's radiation pattern and near field without any external feed excitation. So, we can search for a suitable structure that meets the requirement by analyzing its characteristic field. Figure 2 shows the modal Significance (MS) values of the first three characteristic modes of the metal strip (the length of metal strip is chosen as half-wavelength of $915 \mathrm{MHz}$ ), characteristic current distribution, and the characteristic near magnetic field associated with these modes (the relative dielectric constant of PET material is 4). Figure 3a-c show the vector distribution of the characteristic current corresponding to the maximum MS value of mode 1 to mode 3 and the corresponding $\mathrm{Z}$ component $\left(\mathrm{H}_{\mathrm{Z}}\right)$ of the characteristic magnetic field at $\mathrm{Z}=1 \mathrm{~mm}$ (the positive value is taken in the $+Z$ direction and the negative value is taken in the $-Z$ direction). It is worth to mention here that the strip is placed in xy-plane. The characteristic current of mode 1 shows a similarity with the half-wave mode (associated to $915 \mathrm{MHz}$ ) current of a dipole. Moreover, mode 1 exhibits open characteristic magnetic field distribution, and its normal component has symmetrical distribution about metal strip structure in $\mathrm{Z}=1 \mathrm{~mm}$ plane.

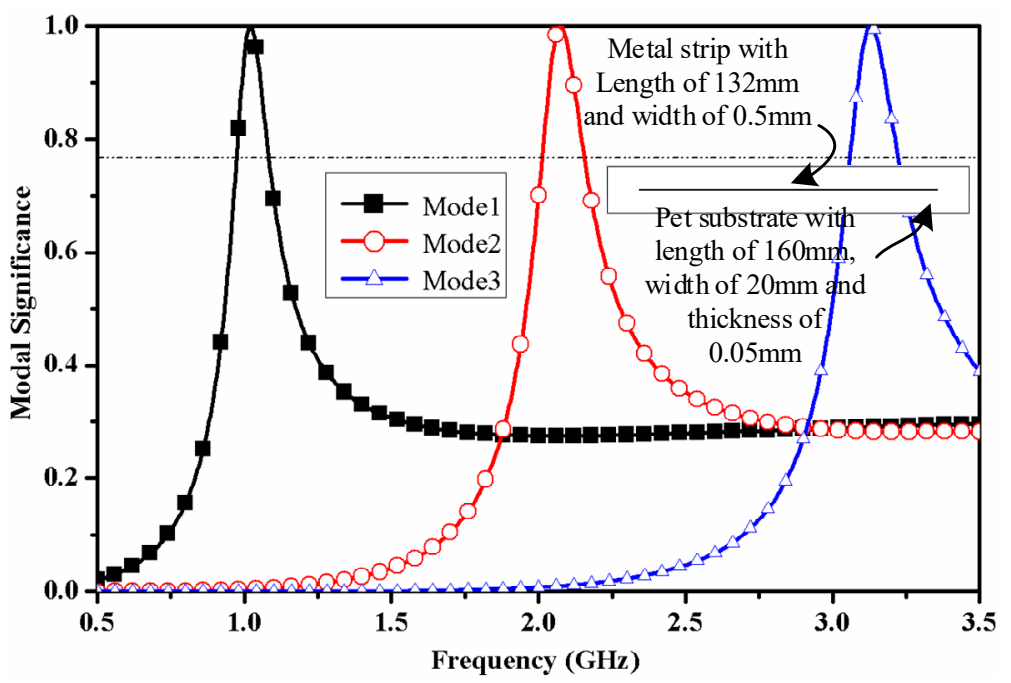

Figure 2. Structure and modal Significance (MS) of metal strip resulted from Characteristics Mode Analysis (CMA).

The characteristic current of mode 2 is the full-wave mode current of the dipole, and its characteristic magnetic field is also open distribution. In the plane $(Z=1 \mathrm{~mm})$, the magnetic field is symmetrically distributed around the metal strip structure. Additionally, the magnetic field value is also symmetrical concerning the long side of the metal strip. The characteristic current of mode 3 shows that the mode current of 1.5 times the wavelength of the dipole. In the plane of $Z=1 \mathrm{~mm}$, the magnetic field lies on both sides along the length of the strip with the same mode value, and opposite direction. Considering the UHF band RFID magnetic field coupled antenna system, the normal distribution of the magnetic field generated by the reader antenna is uniform and in phase in its normal plane. In contrast, the $\mathrm{Z}$ component of the magnetic field (near-field) around the three modes of the metal strip has the same mode value with opposite direction, indicating that the metal strip antenna cannot obtain energy from the magnetic field generated by the reader antenna. 


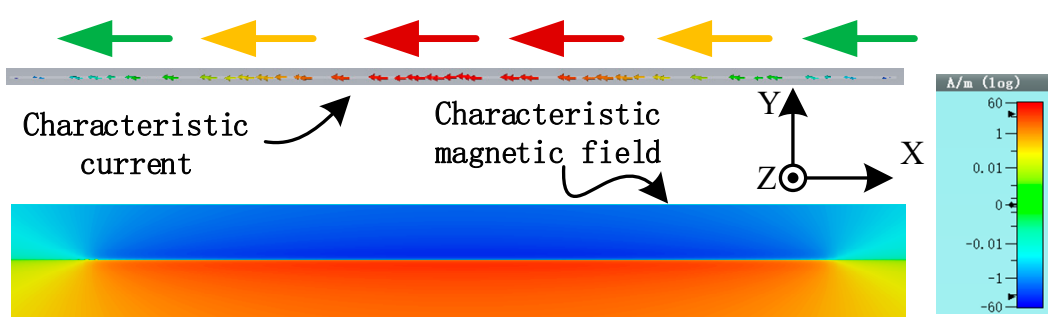

(a)

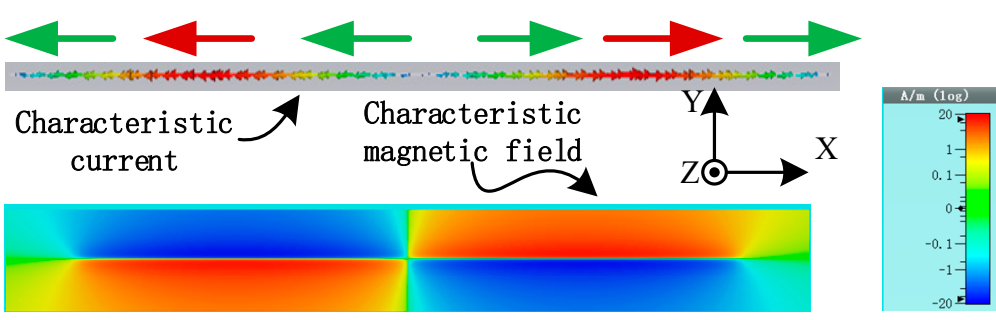

(b)
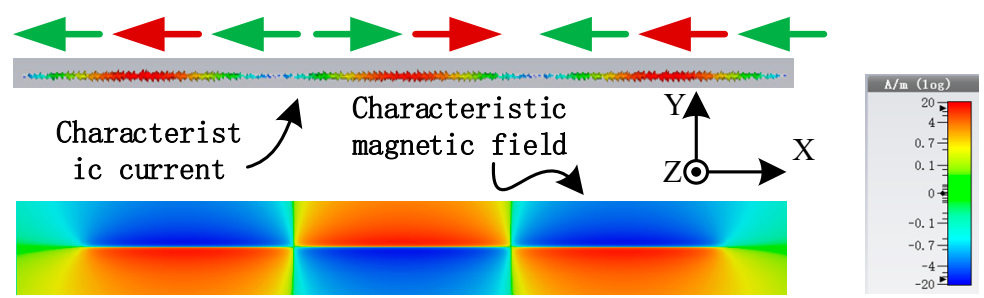

(c)

Figure 3. Characteristics mode current and associated magnetic field profile of metal strip (observed at frequency with maximum MS value) (a) mode 1 (b) mode 2 (c) mode 3.

Figure 4 shows the MS values of the first three characteristic modes of the closed-loop, the distribution of the characteristic current and the magnetic field characteristics (the relative dielectric constant of PET material is 4). Figure 5a-c show the vector distribution of characteristic current at $800 \mathrm{MHz}$ and $\mathrm{Z}$ component of the characteristic magnetic field at $\mathrm{z}=1 \mathrm{~mm}$ for mode 1 to mode 3 . The MS values of mode 1 and mode 2 of the closed metal ring reach the maximum at $800 \mathrm{MHz}$, and it can be seen from the current distribution that mode 1 and mode 2 are a pair of degenerate modes. It can be seen from Figure $5 a, b$ that the magnetic field distribution of mode 1 and mode 2 in the closed metal ring is symmetrical about a midline, with the same amplitude and opposite direction. Therefore, the total magnetic flux in the closed metal ring is zero, since the two modes cannot obtain energy from the uniformly distributed normal magnetic field. Looking at Figure $5 c$, the current flow direction in mode 3 is not reversed, and the values are equal. Therefore, the magnetic field distribution in the closed-loop is in phase, and the corresponding magnetic field on concentric circles is the same. Considering that, in the UHF band RFID magnetic field coupled antenna system, the magnetic field distribution generated by the reader antenna is uniform and in phase in its normal plane. If only focusing on the field distribution, mode 3 can obtain energy from the magnetic field generated by the transmitting coil. However, the MS value corresponding to mode 3 is very small in the whole frequency band. If the excitation is introduced, the current flow direction cannot be reversed in the small electric size. Therefore, for the closed ring structure, it is not possible to expand the size of the closed-loop without changing the current flow direction. Moreover, it is convenient to make a good conjugate matching in such a small size. 


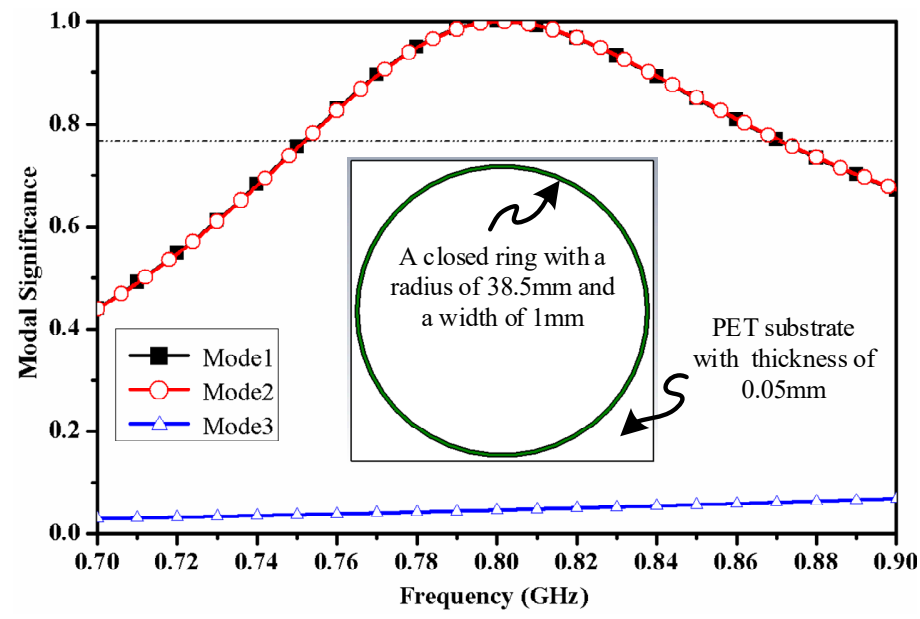

Figure 4. Structure and modal Significance (MS) of closed metal ring resulted from Characteristics Mode Analysis (CMA).
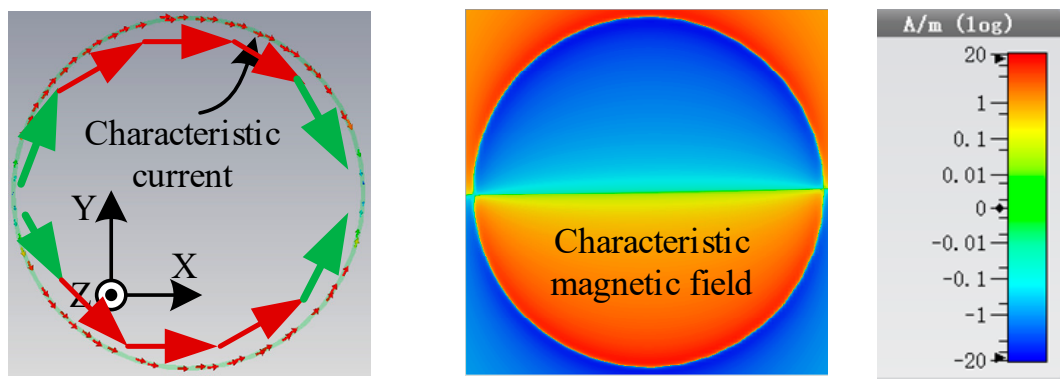

(a)
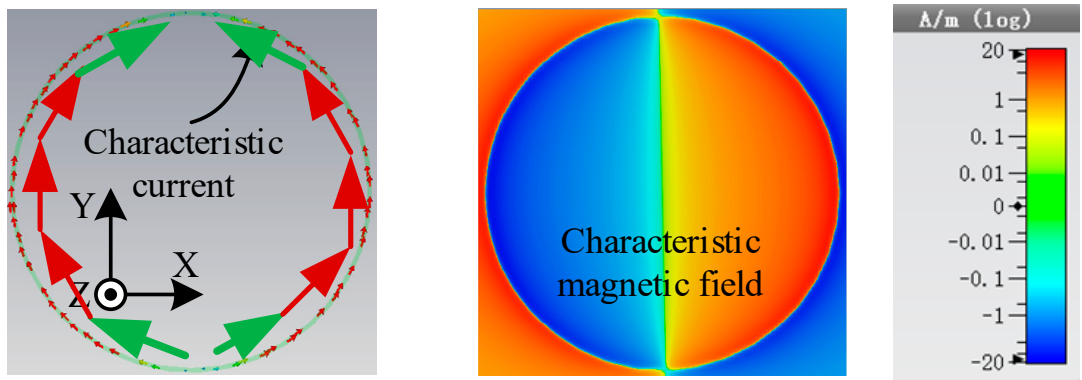

(b)
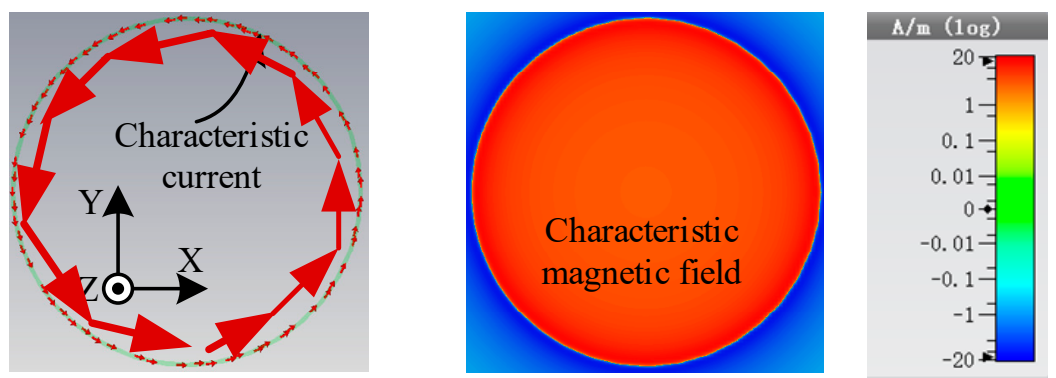

(c)

Figure 5. Characteristics mode current and associated magnetic field profile of closed metal ring (a) mode 1 (b) mode 2 (c) mode 3. 
For choosing electrically small structure, we run CMA for open loop structure with overall length of $100 \mathrm{~mm}$. After running some rigorous simulation tests, a structure with $105 \mathrm{~mm}$ was selected for demonstrating required performance at $1.5 \mathrm{GHz}$. The open structure dimensions were taken by folding $105 \mathrm{~mm}$ length wire to the center line. Figure 6 shows the MS values of the first characteristic mode of the open-loop structure. The proposed open-loop structure shows a peak MS performance at $1.5 \mathrm{GHz}$. Similarly, Figure 7 shows the distribution of the characteristic current and associated characteristic magnetic field (the relative dielectric constant of PET material is 4). In mode 1, MS reaches the maximum at $1.5 \mathrm{GHz}$. By observing the characteristic magnetic field distribution of mode 1, it can be concluded that the near-field magnetic field distribution of this structure is similar to that of mode 3 of the closed-loop. Therefore, the open loop structure has the ability to receive energy from the near-field magnetic field.

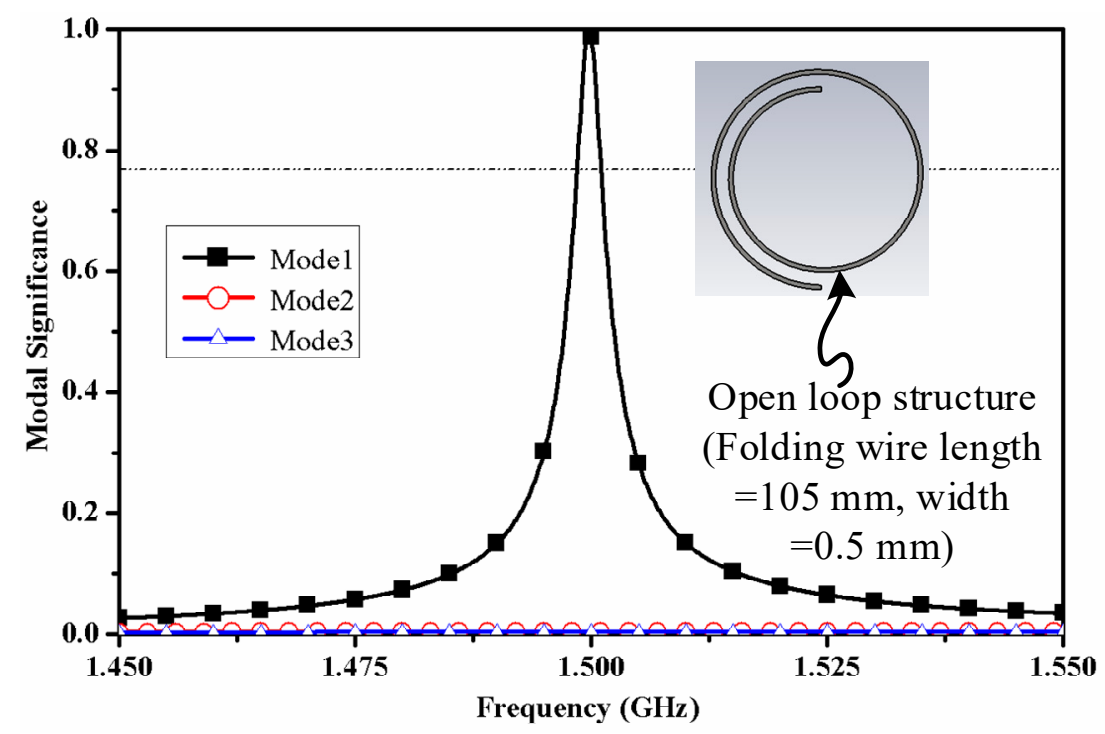

Figure 6. Structure and modal Significance (MS) of Open-loop ring structure resulted from Characteristics Mode Analysis (CMA).
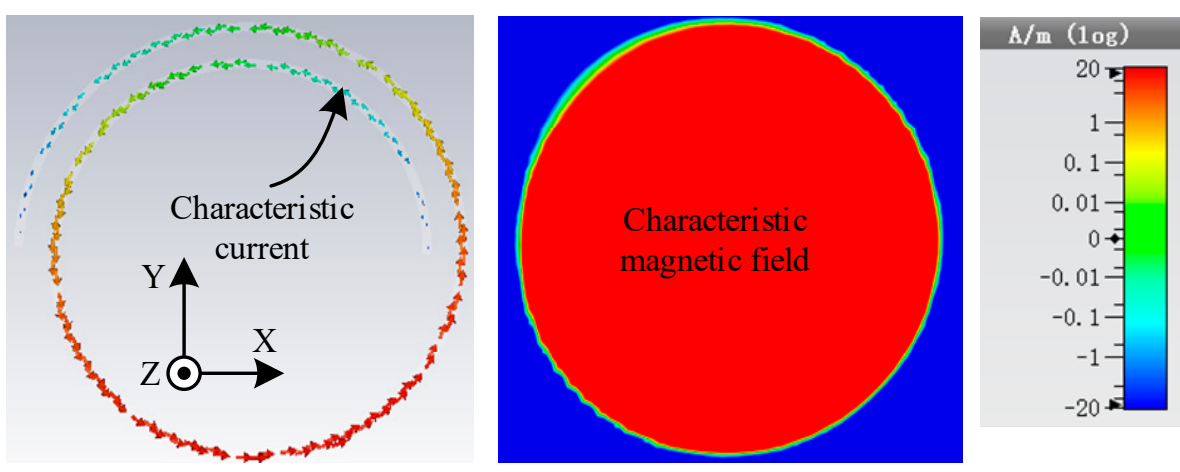

Figure 7. Characteristics mode current and associated magnetic field profile of open-loop ring structure for mode 1 .

\section{Tag Antenna Optimization Using PSO}

The magnetic field intensity of the tag antenna is related to the current intensity. Therefore, we can optimize the uniformity of the magnetic field by optimizing the superposition of the current at different angles. In order to optimize the uniformity of the current, the PSO algorithm is used. Equation (1) shows the mathematical expression of the logarithmic spiral in polar coordinates. From Equation (1), we can know that three parameters $\left(a, b\right.$, and $\left.\theta_{\max }\right)$ can be optimized. However, if the total length (L) of the logarithmic spiral 
is determined, the relationship between the three parameters will also be determined. Equations (2) and (3) show the relationship between the three parameters and the current expression with the $\theta$ as a parameter, respectively. For fitness function $(Q)$, we define a new parameter $I_{\text {angle }}=I_{\theta}+I_{\theta+2 \pi}$, noticeably, if the $\theta+2 \pi \geq \theta_{\max }$, the $I_{\theta+2 \pi}$ is 0 . So, the square difference of the $I_{\text {angle }}$ is the fitness function, which is shown in Equation (4). The $\mathrm{I}_{\text {angle, } \mathrm{I}}$ represents the current value in the 4 th point. The parameter $\mathrm{n}$ indicates the division accuracy from 0 to $2 \pi$.

$$
\begin{gathered}
r=a e^{b \theta}\left(0 \leq \theta \leq \theta_{\max }\right) \\
L=\frac{a}{b}\left(e^{b \theta_{\max }}-1\right) \\
I=\sin \left[\frac{\pi b \theta}{\ln \left(1+\frac{b L}{a}\right)}\right]\left(0 \leq \theta \leq \theta_{\max }\right) \\
Q=\sum_{i=1}^{n}\left(I_{\text {angle }, i}-\frac{\sum_{i=1}^{n} I_{\text {angle }, i}}{n}\right)^{2}
\end{gathered}
$$

Figure 8 shows the flowchart of the proposed design methodology. In the first step, we give the overall length of the logarithmic spiral, and the PSO algorithm was utilized to define the structure for the uniform magnetic field in the normal plane. The PSO algorithm was implemented in MATLAB for achieving optimal tag design parameters to achieve uniform magnetic field performance. Figure $9 a, b$ illustrates the fitness Curve of PSO-based optimization for the proposed near-field tag and the optimized tag parameters achieved after implementing the PSO algorithm in MATLAB, respectively. The optimal tag parameters achieved using the PSO algorithm was utilized further to construct the tag structure in CST Microwave Studio 2018.

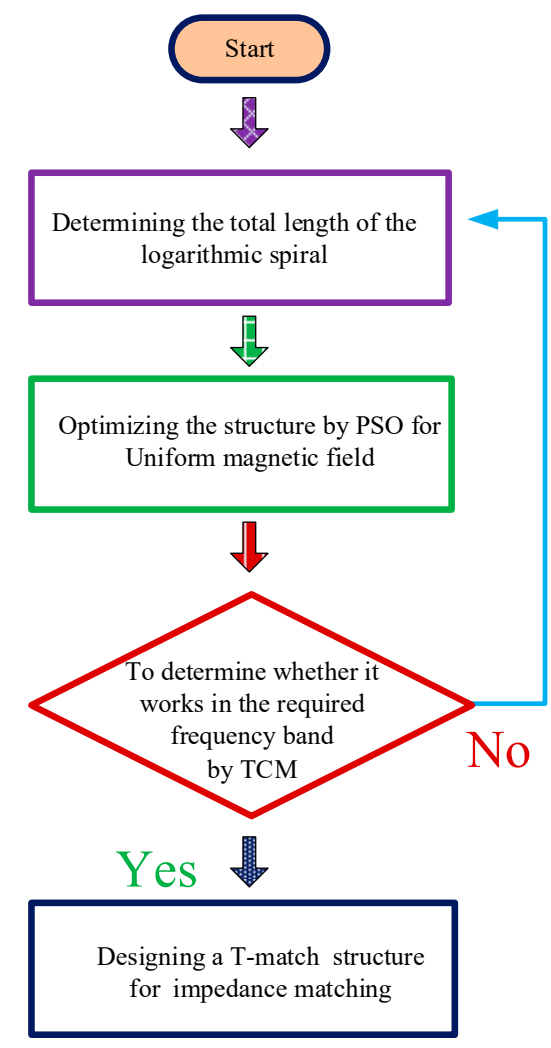

Figure 8. The flowchart of proposed design methodology for optimization of tag. 


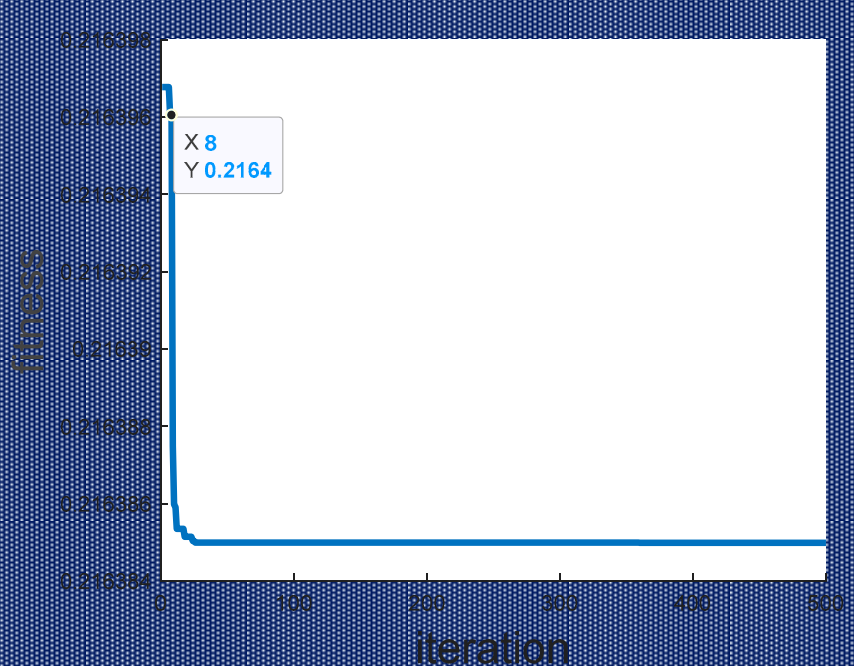

(a)

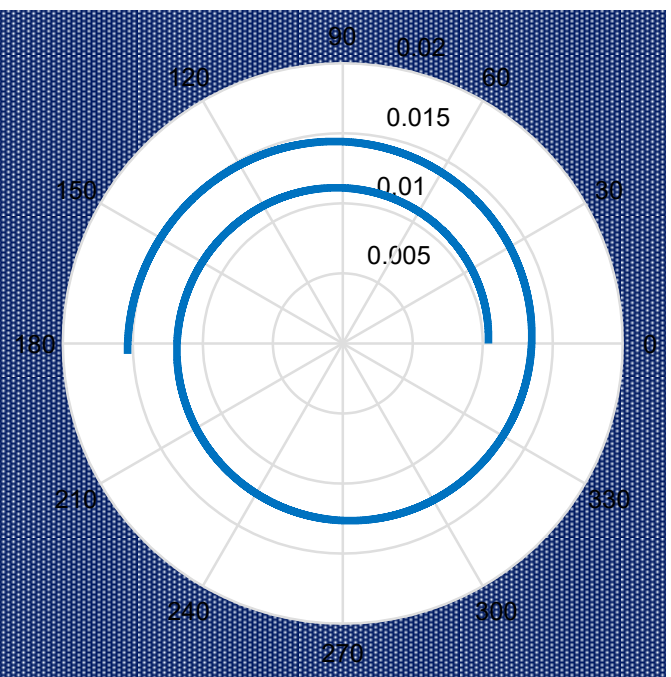

(b)

Figure 9. (a) The fitness Curve of PSO-based optimization for proposed near-field tag (b) The optimized tag parameters achieved after implementing PSO algorithm in MATLAB.

In the second step, the structure using these parameters was calculated by TCM theory to determine whether it has reception capability in the required frequency band. After finalizing the design resulted from TCM, a T-match matching structure was designed, at the maxima of characteristic current, to meet the conjugate match with RFID chip impedance. In case, if design resulted from TCM does meet the requirement, we need to change the overall length and repeat the first step.

Figure 10 shows the structure of the proposed antenna, which consists of a logarithmic spiral and T-match structure. Table 1 gives the detailed parameters of the proposed design. To compare the performance, we chose the same antenna material, substrate, and chip (Monza 4 QT with impedance 11-j143 $\Omega$ ) as the commercial tag. The substrate is PET material (relative permittivity is about 4 ) with a thickness of $0.05 \mathrm{~mm}$. The antenna was printed using aluminum ink. The width of every line in this structure is $0.5 \mathrm{~mm}$. As mentioned above, the excitation should place at the maximum of the characteristic current point, which can be estimated as $\theta=\theta_{\mathrm{d}}$. For the T-match matching structure, it should be flat to avoid influencing the characteristic mode of the logarithmic spiral.

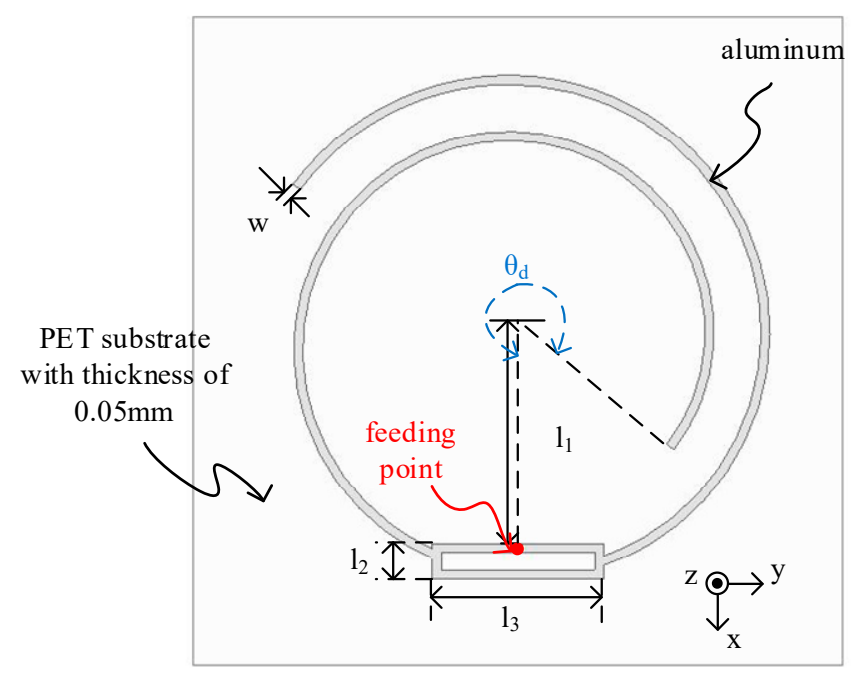

Figure 10. Structure and dimensions of the proposed logarithmic near-field RFID tag. 
Table 1. Dimension of the proposed near-field RFID tag design.

\begin{tabular}{cccc}
\hline Parameter & Dimensions $(\mathbf{m m})$ & Parameter & Dimensions (mm) \\
\hline $\mathrm{a}$ & 0.0103 & $\mathrm{~b}$ & 0.0414 \\
$\mathrm{l}_{1}$ & $11.25 \mathrm{~mm}$ & $\mathrm{l}_{2}$ & $2 \mathrm{~mm}$ \\
$\mathrm{~W}$ & $0.5 \mathrm{~mm}$ & $\theta_{\mathrm{d}}$ & $305 \mathrm{deg}$ \\
$\mathrm{l}_{3}$ & $9.5 \mathrm{~mm}$ & $\theta_{\max }$ & $3 \pi$ \\
\hline
\end{tabular}

\section{Results and Discussion}

Figure 11a shows the MS of proposed open loop structure that shows superior performance of mode 1 in term of significant mode and radiation capacity. The real and imaginary part of impedance associated with the proposed tag antenna is shown in Figure 11b. This tag design shows a good impedance match with the Impinj Monza 4 QT RFID chip with approximate impedance 11-j143 $\Omega$. Figure 12 shows the current distribution and magnetic field $\left(\mathrm{H}_{\mathrm{z}}\right)$ in the normal plane with height $(\mathrm{h}=5 \mathrm{~mm})$ at $915 \mathrm{MHz}$. The current distribution shows, the current remains in phase through the whole tag structure that further leads to the uniform magnetic field in an area equal to the size of the tag. A full-wave electromagnetic simulation has been carried out by means of the commercial software CST Microwave Studio Suite 2018 to prove the performance in terms of current, and magnetic field $\left(\mathrm{H}_{\mathrm{z}}\right)$. To verify the performance of the proposed tag further, a prototype of the proposed tag, along with some other and commercial tags, was fabricated to compare their performance.

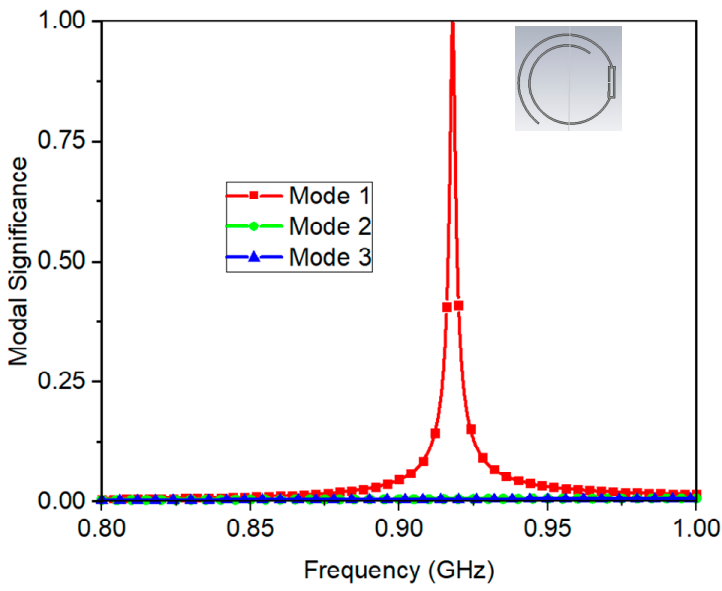

(a)

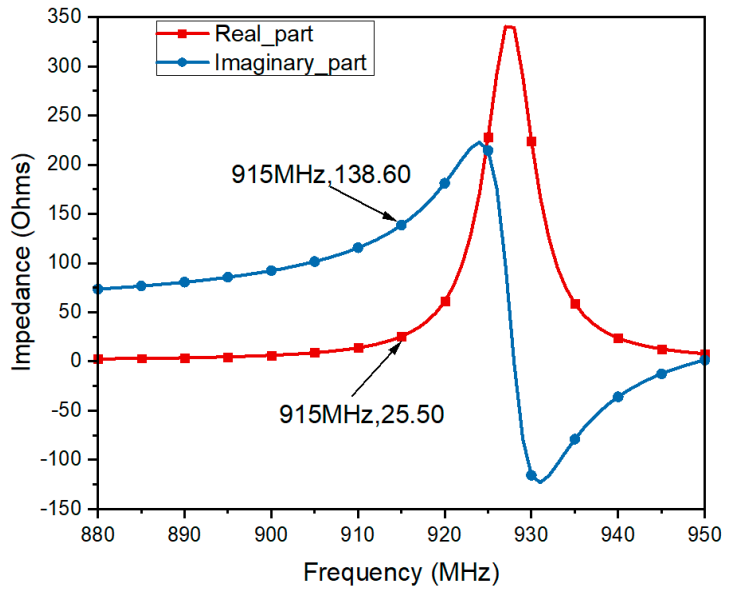

(b)

Figure 11. (a) Modal significance plot of proposed tag structure (b) The simulated real and imaginary impedance plot of proposed logarithmic near-field tag design. 


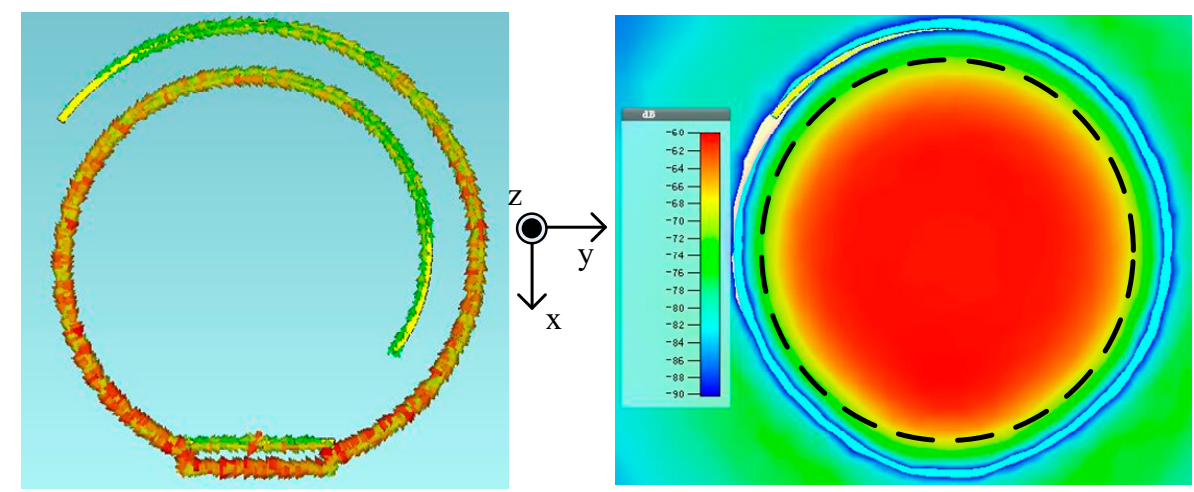

Figure 12. The simulated current distribution and magnetic field $\left(\mathrm{H}_{\mathrm{Z}}\right)$ in the normal plane of proposed logarithmic near-field tag design.

The tag antenna was fabricated using silver nanoparticle paste from Harima NPS series and Fujifilm Dimatix DMP-2831 inkjet printer with a 10 pL volume cartridge. Moreover, the tag was fabricated on $50 \mu \mathrm{m}$ PET substrate with 4 layered printing approach.

Figure 13 shows five tested tags, which are numbered as tag1 to tag5. Tag1 is our proposed tag, and tag2 is the commercial button tag, which has the electric-small loop. The tag3, tag4, and tag5 are printed in the PCB substrate, and the chips of them are all H4 SOT packaging. Tag3 is based on the same theory as the proposed tag in this paper. The antenna of tag 4 and tag 5 are both full-wavelength loops, and tag 4 is the miniaturization of tag5. A near-field antenna in the published paper is used as the reader antenna [5] to test the performance of the five tags. Figure 14 shows the test environment, which is a semi-open microwave anechoic chamber. The setup was explored using different transmit power levels for testing read range and further specifying the integration zone. The maximum read distance, which is tested by the center to center of the tested tag and the reader antenna is recorded in Table 2 .

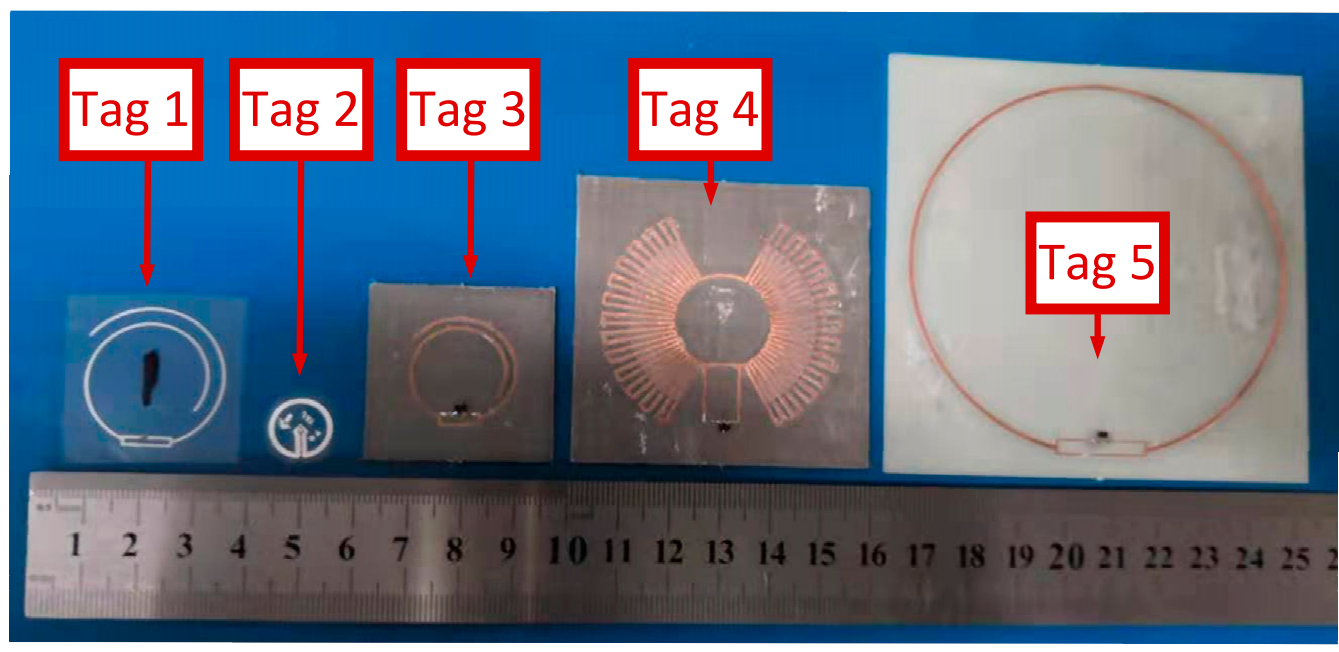

Figure 13. Fabricated prototype of proposed tag (Tag1) along with some other tags fabricated for comparison proposes. 


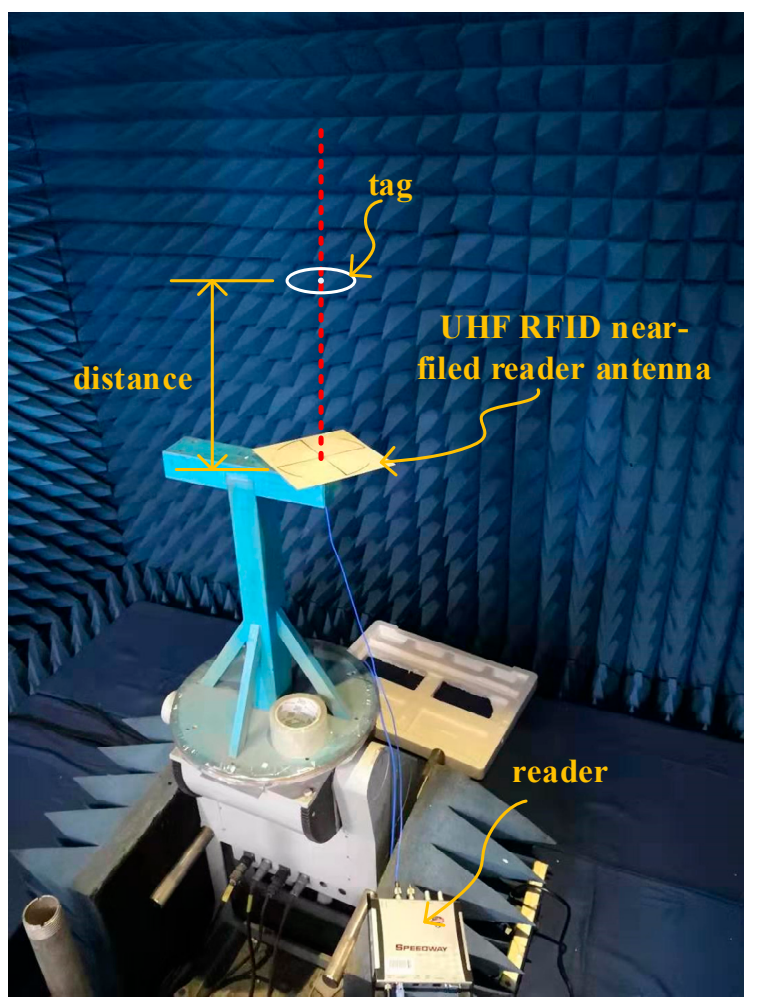

Figure 14. Read range testing environment setup in a semi-open microwave anechoic chamber.

Table 2. Comparison of read range and interrogation zone of proposed with other tag designs.

\begin{tabular}{cccccc}
\hline Transmit Power & $\begin{array}{c}\text { Tag1 } \\
\text { (Proposed Tag) }\end{array}$ & $\begin{array}{c}\text { Tag2 } \\
\text { (Commercial Tag) }\end{array}$ & Tag3 & Tag4 & Tag5 \\
\hline $10 \mathrm{dBm}$ & $13 \mathrm{~cm}$ & $4 \mathrm{~cm}$ & $18 \mathrm{~cm}$ & $1 \mathrm{~cm}$ & $1 \mathrm{~cm}$ \\
\hline $15 \mathrm{dBm}$ & $19 \mathrm{~cm}$ & $8 \mathrm{~cm}$ & $23 \mathrm{~cm}$ & $2 \mathrm{~cm}$ & $2 \mathrm{~cm}$ \\
\hline $20 \mathrm{dBm}$ & $25 \mathrm{~cm}$ & $12 \mathrm{~cm}$ & $30 \mathrm{~cm}$ & $3 \mathrm{~cm}$ & $3 \mathrm{~cm}$ \\
\hline $30 \mathrm{dBm}$ & $46 \mathrm{~cm}$ & $27 \mathrm{~cm}$ & $60 \mathrm{~cm}$ & - & - \\
\hline
\end{tabular}

From Table 2, tag4 and tag5 have the biggest size in all tags, but they are hardly readable, which means it is not the bigger size, the more read distance for the closed-loop antenna. Tag1 has three times more read distance as compared to tag2, which means that a closed structure is not always necessary for receiving near field energy in the case of the UHF band. Furthermore, the proposed tag opened structure can provide both the flexibility of extending the receiving area as well as adjust the uniformity of the magnetic field, which is more suitable in this circumstance. Because of the differences in sensitivity (the sensitivity of Monza QT is $-17.4 \mathrm{dBm}$ and the $\mathrm{H} 4 \mathrm{SOT}$ is $-20.5 \mathrm{dBm}$ ), the read distance of tag3 is more than tag1. The test results validate our proposed design very well.

Finally, the proposed tag antenna was used experimentally for item-level tagging of jewelry products, as shown in Figure 15. The setup consisted of a foam-based frame, nearfield reader antenna, RFID reader, and tagged jewelry products. The proposed tag/sensor exhibited $100 \%$ reading accuracy up to $46 \mathrm{~cm}$ with transmit power of $30 \mathrm{dBm}$. In addition to this, the proposed tag antenna can be exploited for temperature sensing of the human body as its main working principle is based on the magnetic field that is significantly less affected by permittivity changes. 


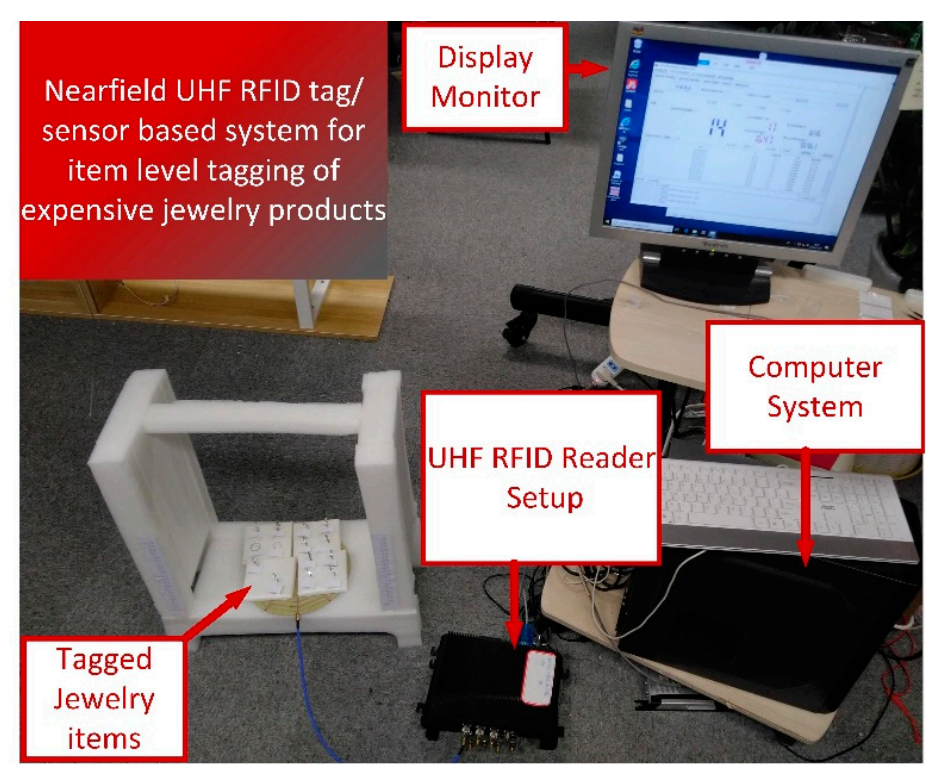

Figure 15. Experimental testing regarding item-level tagging of jewelry products using proposed tag/sensor.

\section{Conclusions}

This paper presents a novel structure UHF RFID tag based on a combination of PSO and TCM. As compared to traditional electric-small near-field tags, the proposed tag design uses the logarithmic spiral as the radiating structure. The benefit of the logarithmic spiral structure lies in its large magnetic field receiving area that can be extended to reach a higher limit reading distance. The combination of PSO and TCM is used to get the uniform magnetic field and desired resonant frequency. Five tags are tested to validate our opinion, and compared with the frequently-used commercial near-field tag (Impinj J41), our proposed design can achieve three times more read distance with $10 \mathrm{dBm}$ transmission power. This tag can reach an ideal read distance under a small transmitting power, which greatly reduces the possibility of far-field misreading under high transmitting power conditions. On the one hand, the design greatly relieves the pressure on reader antenna designers, and its read distance can further increase by using the latest chip. Furthermore, it gives a new idea for UHF RFID near-field tag design, which does not base on commonly used closed electric-small loop structure. For commercial item-level applications, this novel near field tag shows great potential towards practical near field UHF applications.

Author Contributions: Conceptualization, A.S., Y.Y. and J.O.; methodology, A.S., Y.Y. and Q.H.A.; software, A.S., Y.Y. and J.O.; validation, Q.H.A., M.A.I. and K.A. (Kamran Arshad); formal analysis, J.O., Q.H.A., M.A.I.; resources, K.A. (Kamran Arshad), K.A. (Khaled Assaleh) and M.A.I.; writingoriginal draft preparation, Y.Y., A.S.; writing—review and editing, Q.H.A., H.T.C., A.A.A., T.A. and N.R.; visualization, J.O., H.T.C., N.R.; supervision, J.O., Q.H.A. and M.A.I. All authors have read and agreed to the published version of the manuscript.

Funding: This work was supported in part by Ajman University Internal Research Grant and in part by Taif University Research Grant under Project TURSP-2020/277.

Acknowledgments: We would like to thank antenna wireless experts for their help regarding tag fabrication and testing. Also, we would like to thank the Ajman University Internal Research Grant for partially supporting this work.

Conflicts of Interest: The authors declare no conflict of interest. 


\section{References}

1. Escobedo, P.; Bhattacharjee, M.; Nikbakhtnasrabadi, F.; Dahiya, R. Smart bandage with wireless strain and temperature sensors and batteryless NFC tag. IEEE Internet Things J. 2021, 8, 5093-5100. [CrossRef]

2. Sharif, A.; Ouyang, J.; Yang, F.; Chattha, H.T.; Imran, M.A.; Alomainy, A.; Abbasi, Q.H. Low-cost inkjet-printed UHF RFID tag-based system for internet of things applications using characteristic modes. IEEE Internet Things J. 2019, 6, $3962-3975$. [CrossRef]

3. Beliatis, M.J.; Jensen, K.; Ellegaard, L.; Aagaard, A.; Presser, M. Next generation industrial IoT digitalization for traceability in metal manufacturing industry: A case study of industry 4.0. Electronics 2021, 10, 628. [CrossRef]

4. Jabeen, I.; Ejaz, A.; Rahman, M.U.; Naghshvarianjahromi, M.; Khan, M.J.; Amin, Y.; Tenhunen, H. Data-dense and miniature chipless moisture sensor RFID tag for internet of things. Electronics 2019, 8, 1182. [CrossRef]

5. Martínez-Olmos, A.; Salmerón, J.F.; Ruiz, N.L.; Torres, A.R.; Capitan-Vallvey, L.F.; Palma, A.J. Screen printed flexible radiofrequency identification tag for oxygen monitoring. Anal. Chem. 2013, 85, 11098-11105. [CrossRef]

6. $\quad$ Escobedo, P.; Erenas, M.M.; López-Ruiz, N.; Carvajal, M.A.; Gonzalez-Chocano, S.; de Orbe-Payá, I.; Capitán-Valley, L.F.; Palma, A.J.; Martínez-Olmos, A. Flexible passive near field communication tag for multigas sensing. Anal. Chem. 2017, 89, 1697-1703. [CrossRef] [PubMed]

7. Escobedo, P.; Carvajal, M.A.; Capitán-Vallvey, L.F.; Salmerón, J.F.; Martínez-Olmos, A.; Palma, A.J. Passive UHF RFID tag for multispectral assessment. Sensors 2016, 16, 1085. [CrossRef]

8. Sharif, A.; Ouyang, J.; Yang, F.; Long, R.; Ishfaq, M.K. Tunable platform tolerant antenna design for RFID and IoT applications using characteristic mode analysis. Wirel. Commun. Mob. Comput. 2018, 2018, 1-10. [CrossRef]

9. Sharif, A.; Ouyang, J.; Yan, Y.; Raza, A.; Imran, M.A.; Abbasi, Q.H. Low-cost inkjet-printed RFID tag antenna design for remote healthcare applications. IEEE J. Electromagn. RF Microw. Med. Biol. 2019, 3, 261-268. [CrossRef]

10. Althobaiti, T.; Sharif, A.; Ouyang, J.; Ramzan, N.; Abbasi, Q.H. Planar pyramid shaped UHF RFID tag antenna with polarisation diversity for IoT applications using characteristics mode analysis. IEEE Access 2020, 8, 103684-103696. [CrossRef]

11. Morgado, T.A.; Alves, J.M.; Marcos, J.; Maslovski, S.I.; Costa, J.R.; Fernandes, C.A.; Silveirinha, M.G. Spatially confined UHF RFID detection with a metamaterial grid. IEEE Trans. Antennas Propag. 2014, 62, 378-384. [CrossRef]

12. Lin, Y.; Liao, C.; Chen, H.; Jiang, Z. Compact folded square-loop antenna for reading near-field RFID tags in blood sample tracking system. Electron. Lett. 2017, 53, 1627-1628. [CrossRef]

13. Zeng, Y.; Chen, Z.N.; Qing, X.; Jin, J.-M. A directional, closely spaced zero-phase-shift-line loop array for UHF near-field RFID reader antennas. IEEE Trans. Antennas Propag. 2018, 66, 5639-5642. [CrossRef]

14. Wei, X.-D.; Zhang, H.-L.; Hu, B.-J. Novel broadband center-fed UHF Near-Field RFID reader antenna. IEEE Antennas Wirel. Propag. Lett. 2015, 14, 703-706. [CrossRef]

15. Hongwei, S.; Huijun, C.; Yuhui, H. A mini UHF tag with on chip antenna based on gold bump process. In Proceedings of the 12th IEEE International Conference on Solid-State and Integrated Circuit Technology (ICSICT), Guilin, China, 28-31 October 2014. [CrossRef]

16. Dong, J.; Li, X. UHF near-field tags design based on split ring resonator. In Proceedings of the Asia-Pacific Microwave Conference Proceedings, APMC, Melbourne, VIC, Australia, 5-8 December 2011; pp. 1794-1797.

17. Kim, S.-Y.; Ahn, S.-H.; Lee, W.-S. Near-field UHF quadruple loop antenna with uniform field distribution for item-level tagging. IEEE Antennas Wirel. Propag. Lett. 2021, 20, 523-527. [CrossRef]

18. Gao, Y.; Ma, R.; Zhang, Q.; Parini, C. Design of very-low-profile circular UHF small antenna using characteristic mode analysis IET Microw. Antennas Propag. 2017, 11, 1113-1120. [CrossRef]

19. Robinson, J.; Rahmat-Samii, Y. Particle swarm optimization in electromagnetics. IEEE Trans. Antennas Propag. 2004, 52, 397-407. [CrossRef]

20. Rahmat-Samii, Y.; Kovitz, J.M.; Rajagopalan, H. Nature-inspired optimization techniques in communication antenna designs Proc. IEEE 2012, 100, 2132-2144. [CrossRef]

21. Zaharis, Z.D.; Gravas, I.P.; Lazaridis, P.; Glover, I.A.; Antonopoulos, C.S.; Xenos, T.D. Optimal LTE-protected LPDA design for DVB-T reception using particle swarm optimization with velocity mutation. IEEE Trans. Antennas Propag. 2018, 66, $3926-3935$. [CrossRef]

22. Li, Y.-L.; Shao, W.; You, L.; Wang, B.-Z. An improved PSO algorithm and its application to UWB antenna design. IEEE Antennas Wirel. Propag. Lett. 2013, 12, 1236-1239. [CrossRef]

23. Alnas, J.; Giddings, G.; Jeong, N. Bandwidth improvement of an inverted-f antenna using dynamic hybrid binary particle swarm optimization. Appl. Sci. 2021, 11, 2559. [CrossRef]

24. Simone, M.; Fanti, A.; Lodi, M.; Pisanu, T.; Mazzarella, G. An in-line coaxial-to-waveguide transition for Q-band single-feed-perbeam antenna systems. Appl. Sci. 2021, 11, 2524. [CrossRef]

25. Kibria, S.; Islam, M.T.; Yatim, B. New compact dual-band circularly polarized universal RFID reader antenna using ramped convergence particle swarm optimization. IEEE Trans. Antennas Propag. 2014, 62, 2795-2801. [CrossRef]

26. Di Giampaolo, E.; Fornì, F.; Marrocco, G. RFID-network planning by particle swarm optimization. In Proceedings of the Fourth European Conference on Antennas and Propagation, Barcelona, Spain, 12-16 April 2010; pp. 1-5.

27. Yan, Y.; Ouyang, J.; Ma, X.; Wang, R.; Sharif, A. Circularly polarized rfid tag antenna design for metallic poles using characteristic mode analysis. IEEE Antennas Wirel. Propag. Lett. 2019, 18, 1327-1331. [CrossRef] 
28. Kim, S. Inkjet-Printed Electronics on Paper for RF Identification (RFID) and Sensing. Electronics 2020, 9, 1636. [CrossRef]

29. Han, P.; Zhang, Z.; Xia, Y.; Mei, N. A 920-MHz dual-mode receiver with energy harvesting for UHF RFID tag and IoT. Electronics 2020, 9, 1042. [CrossRef]

30. Andrade, L.; Figueiredo, J.; Tlemçani, M. A New RFID-Identification strategy applied to the marble extraction industry. Electronics 2021, 10, 491. [CrossRef] 